\title{
Letter \\ Power Efficient Current Driver Based on Negative Boosting for High-Speed Lasers
}

\author{
Saad Arslan ${ }^{1,2}{ }^{(}$, Syed Asmat Ali Shah ${ }^{1,3}$ and HyungWon Kim ${ }^{1, *(\mathbb{D}}$ \\ 1 Department of Electronics Engineering, Chungbuk National University, Chungdae-ro 1, Seowon-gu, \\ Cheongju 28644, Korea; saad@cbnu.ac.kr (S.A.); syed@cbnu.ac.kr (S.A.A.S.) \\ 2 Department of Electrical and Computer Engineering, COMSATS University Islamabad, Park Road, \\ Tarlai Kalan, Islamabad 45550, Pakistan \\ 3 Department of Electrical and Computer Engineering, COMSATS University Islamabad, Abbottabad \\ Campus, University Road, Tobe Camp, Abbottabad 22060, Pakistan \\ * Correspondence: hwkim@cbnu.ac.kr
}

Received: 30 September 2019; Accepted: 6 November 2019; Published: 8 November 2019

\begin{abstract}
Vertical-cavity surface-emitting lasers (VCSELs) are commonly used in high-speed optical communication and 3D sensing applications. Both of these applications require high switching frequency and a short rise time of the VCSEL current. The parasitic inductance of the wire (connecting the driver with VCSEL) makes it challenging to achieve a short rise time, which often incur increased supply voltage and excessive power consumption. This paper utilizes a momentary boosting in supply voltage to overcome the parasitic inductance of the wire with minimal power overhead. The proposed technique uses a precalculated boosting capacitance to produce negative voltage for common-anode VCSELs. The boosting capacitance provides the required amount of charge during the rising transition and automatically disconnects itself in steady-state. Circuit simulations reveal up to three times shorter rise time at the negligible cost of less than $10 \%$ power overhead.
\end{abstract}

Keywords: CMOS laser drivers; pulsed laser diode driver; vertical cavity surface emitting lasers (VCSEL); negative supply boosting; switching circuits

\section{Introduction}

Vertical-cavity surface-emitting lasers (VCSELs) are widely used in high-bandwidth optical interconnects [1,2] and 3D sensing applications [3,4]. Optical interconnects are part of the Internet structure for quite some time, meeting the demands of the growing bandwidth requirements. The relatively newer 3D sensing applications either rely on time-of-flight (ToF) or structured light beam for depth measurement. VCSEL-based 3D sensing is getting increasingly common for facial recognition in smartphones and object tracking in automobiles and robots using LiDAR (Light Detection and Ranging). Modern optical interconnects and 3D sensing applications require short-pulsed, high-power driving circuits for VCSELs.

Figure 1 illustrates an example circuit connecting a VCSEL and its driver, emphasizing the parasitic resistance and inductance of the wire. With the required pulse widths (of the VCSELs) approaching one nanosecond or less, it becomes challenging to design its driver circuit [5]. This is because the parasitic components of the wire, connecting VCSEL with the driver, restrict fast transitions in the current. Some advanced methods to reduce parasitics include bonding the driver die directly to the printed circuit board (PCB) [6,7], and bonding the driver and VCSEL dies directly to each other without a PCB $[8,9]$. 


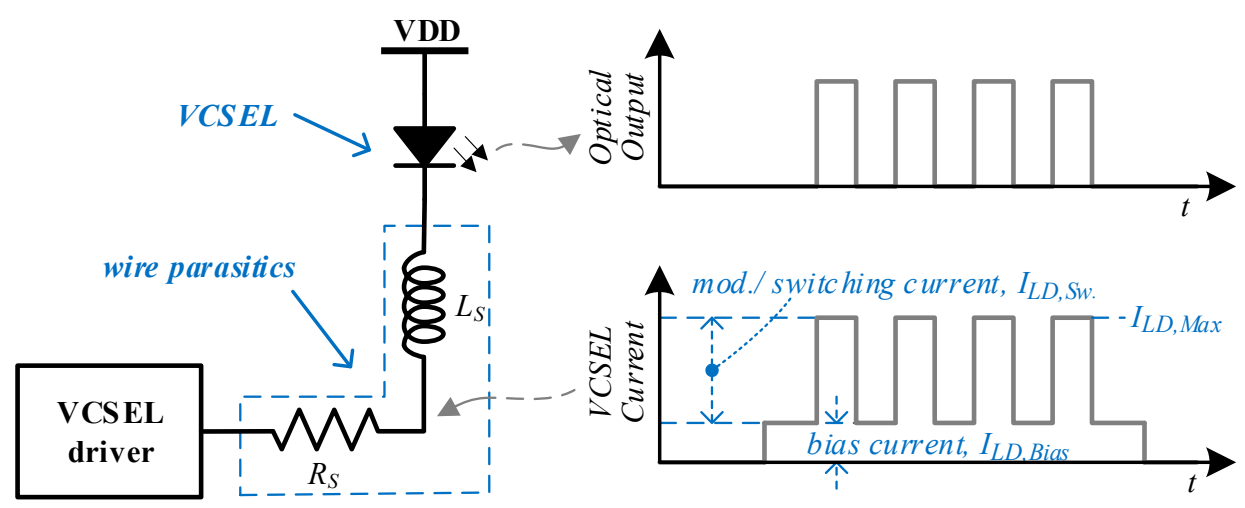

Figure 1. Common Anode (CA) vertical-cavity surface-emitting laser (VCSEL) connection with its driver circuit, in the presence of wire parasitics consisting of resistance and inductance.

The methods in [6-9], however, result in increased cost, and may not be able to meet the transition requirements on their own. Additional methods are therefore needed to ensure sharper transitions to meet the required specifications.

Due to low-cost and low-power requirements, the Complementary Metal Oxide Semiconductor (CMOS) is a suitable choice for the implementation of the driver circuits [10]. Also, it is strongly recommended to use an integrated driver circuit which can reduce the wire parasitics and thus allow faster signal transitions [11]. Many drivers use a large supply voltage to achieve the desired rate of change in the current by overcoming the parasitic inductance of the wire $[12,13]$. A large voltage supply, however, results in excessive power consumption, making the driver unsuitable for mobile applications. Some researchers have used an additional external boosting inductor to create a momentary boost in the supply voltage $[10,14]$. For such drivers, additional circuits and wires are required to charge the large boosting inductor, resulting in increased power consumption and area overhead. Therefore, the additional external components make the inductor boosting method less suitable for fully integrated applications.

On the other hand, switched capacitor circuits offer the benefit of on-chip integration, which makes them suitable for mobile applications $[15,16]$. Moreover, with deep submicron processes offering high density on-chip capacitance, high power applications can be addressed. A capacitor boosting technique is presented in [17], which uses a flying capacitor to boost (double) the VDD to have a sharp rising of the VCSEL current. This technique, however, requires many additional wires, and does not support adjustable current levels. In addition, it has extra metal-oxide-semiconductor field-effect transistors (MOSFETs) in the driving current path, posing limitations for high-speed and high-power applications. Additional research is needed to overcome the limitation of the previous drivers for high-power applications, minimize the power overhead and eliminate the needs for external components.

This letter proposes a fully integrated, switched capacitor based, negative supply boosting during the rising transition to overcome the parasitics. The proposed VCSEL driver also supports multiple current levels, with significantly lower power consumption compared to previous boosting methods mentioned above. The proposed driver, being fully integrated and power efficient, targets 3D sensing for mobile platforms as the main application. It is, however, possible to use the driver in a vast number of applications from optical interconnects to biomedical devices.

Section 2 analyzes the rise time of the VCSEL current in presence of the parasitic elements. Section 3 presents the proposed architecture and its analytical model. Section 4 discusses circuit implementation and evaluates the performance of the proposed architecture. Finally, a discussion on the interpretation and usefulness of the simulation results is presented in Section 5 , followed by conclusions in Section 6. 


\section{Analysis of the VCSEL Rise Time}

To explore low power methods to reduce the rise time of the VCSEL current, consider a typical VCSEL connection with the driver circuit shown in Figure 1. There are two types of current flowing through the VCSEL or Laser Diode (LD), which are bias $I_{L D, B i a s}$ and modulation/switching current $I_{L D, S w}$. [1]. During operation, the bias current stays constant while the switching current has a pulsating behavior with high switching frequency. The parasitic inductance of the wire resists sudden changes in the switching current and degrades the rise and fall time, thereby limiting the switching frequency. Due to the presence of these parasitics, it can be challenging to achieve a sub-nanosecond rise time at large switching current levels [5]. The purpose of the bias current is used to overcome the threshold current of the VCSEL, while producing no optical output. Without the bias current, the switching current would have longer transition times, with the total current going from 0 to $I_{L D, M a x}$.

The current flowing through the inductor (and the VCSEL) of Figure 1, during the rising transition, can be calculated by (1).

$$
I_{L D}(t)=\frac{V_{R L}}{R_{S}}\left(1-e^{-t R_{S} / L_{S}}\right),
$$

Here, $V_{R L}$ is the voltage drop across the wire (inductor and resistor), $L_{S}$ is inductance, $R_{S}$ is resistance and $t$ is the time instant. The voltage drop on the wire is expressed by (2).

$$
V_{R L}=V_{D D}-V_{L D}-V_{D r o}
$$

In (2), $V_{D D}$ is the supply voltage, $V_{L D}$ is the forward voltage drop of the VCSEL and $V_{D r v}$ is the voltage drop across the MOSFETs of the driver. To find the rise time $(10 \%$ to $90 \%)$ for a target current $I_{\mathrm{LD}, \mathrm{Max}}$, we can start by re-arranging (1), to get the rise time by (5).

$$
\begin{gathered}
t=-\frac{L_{S}}{R_{S}}\left[\ln \left(V_{R L}-i_{L D}(t) R_{S}\right)-\ln \left(V_{R L}\right)\right], \\
t_{r}=t_{90 \%}-t_{10 \%,} \\
t_{r}=-\frac{L_{S}}{R_{S}} \ln \left(\frac{V_{R L}-0.9 \times I_{L D, M a x} \times R_{S}}{V_{R L}-0.1 \times I_{L D, \operatorname{Max}} \times R_{S}}\right),
\end{gathered}
$$

It is evident from (5) that the rise time of the current can be reduced by reducing the parasitic inductance. Generally, the width of the trace (connecting the driver to the VCESL) is kept minimum, in order to have low parasitic values. To explore additional methods of reducing rise time, we need to investigate the dependency of $t_{r}$ on $V_{R L}$. Figure 2 illustrates the change in rise time over a range of $V_{R L}$ using (5), for $I_{\mathrm{LD}, \mathrm{Max}}$ in the range of $1 \mathrm{~A}$ to $3 \mathrm{~A}$. Figure 2 assumes a typical parasitic model for the bonding wires and PCB traces, $R_{S}=50 \mathrm{~m} \Omega, L_{S}=1 \mathrm{nH}$. It can be observed from Figure 2 that the rise time can be decreased by increasing the voltage across the wire. As expressed in (2), the voltage across the wire can be increased by (1) decreasing $V_{L D}$, (2) decreasing $V_{D r v}$, and (3) by increasing the power supply voltage. Using a larger power supply $[12,13]$ or using a temporary boosted supply $[10,14,17]$ are known to decrease rise time. Another notable observation is that the voltage required across the wire varies with $I_{L D, M a x}$, for the same rise time. Therefore, to avoid overshoot while maintaining the rise time, the voltage drop across the wire must be decreased with the decrease in $I_{\mathrm{LD}, \mathrm{Max}}$.

A conventional driver circuit is shown in Figure 3a with its current waveform in Figure 3d. The external inductor-based voltage boosting method employed in $[10,14]$, which boosts the voltage during the rise time, is shown as a simplified circuit in Figure $3 \mathrm{~b}$. During the off-period $(E N=0)$, the boosting inductor is charged to $I_{\mathrm{LD}, \mathrm{Max}}$, as shown in Figure $3 \mathrm{~d}$. During the on-period $(E N=1)$, the normal path connects, and the boosting inductor momentarily raises voltage on the anode of the VCSEL to force current $I_{\mathrm{LD}, \mathrm{Max}}$. This, however, requires complex driver circuitry, which incurs excessive area overhead in addition to external inductor and wires. Furthermore, the power consumption doubles, since its pre-charge current continuously flows even during the off-period, as demonstrated by 
comparing Figures $3 \mathrm{c}$,d. In contrast, the technique proposed in this paper boosts the supply voltage up to $2 x$ VDD, during rising transition, by creating negative VDD. The proposed technique accomplished a short rise time without any external components or wires.

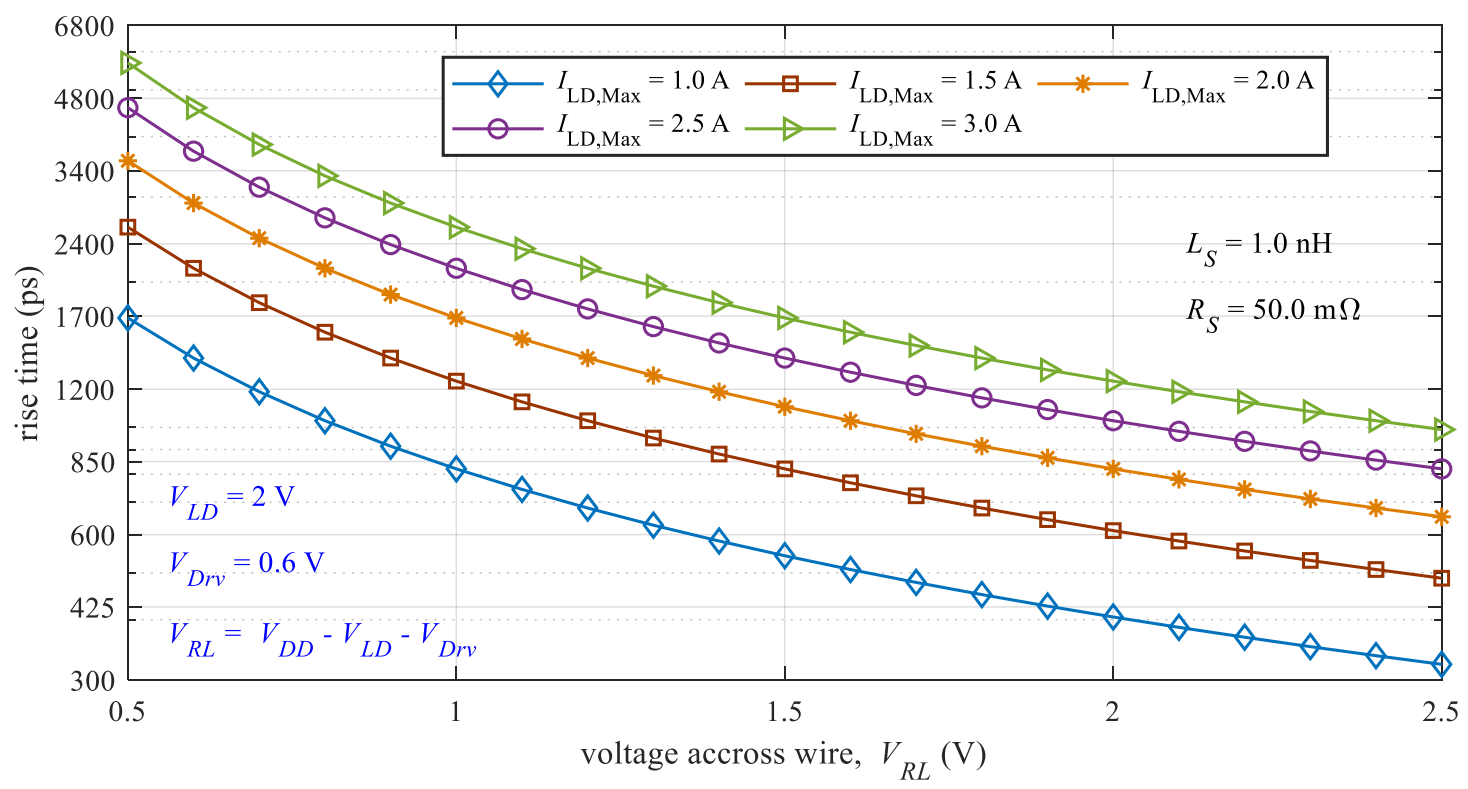

Figure 2. Rise time reduction by increasing voltage across wire.

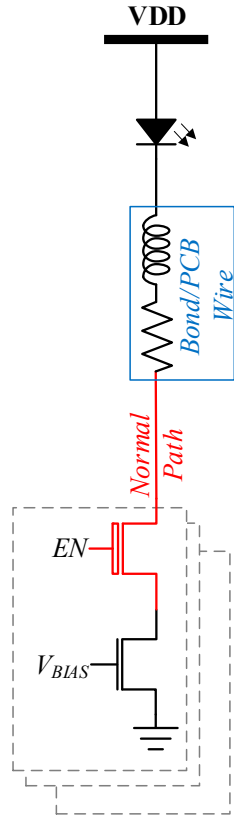

(a)

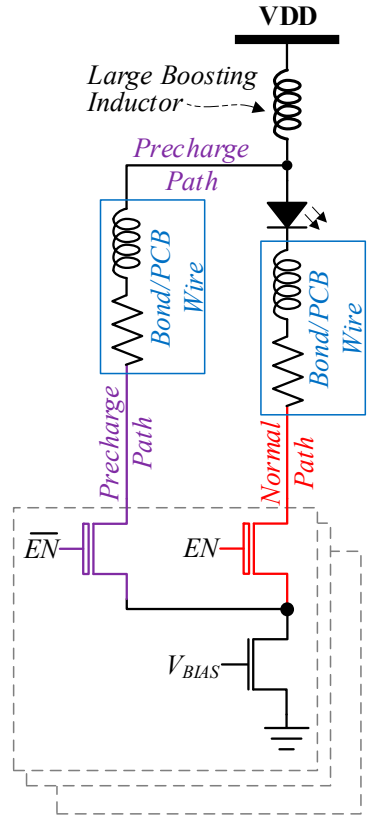

(b)

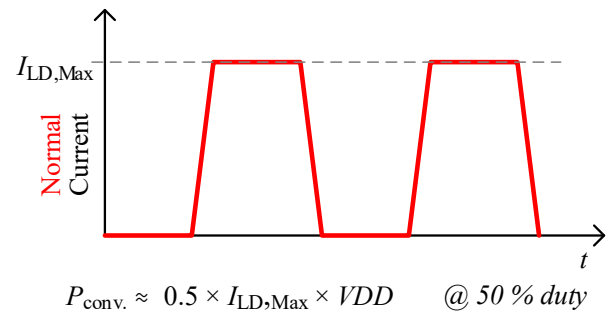

(c)

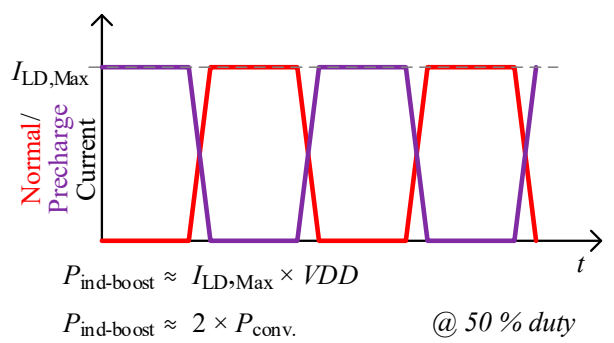

(d)

Figure 3. (a) Conventional driver with VCSEL. (b) Simplistic inductor boosting-based driver with VCSEL. (c) Conventional driver current waveform. (d) Inductor boosting based driver current waveforms.

\section{Negative Voltage Boosting}

To achieve faster rising transition and low power consumption, a precalculated magnitude and duration of the supply voltage boosting is needed. The proposed design is shown in Figure 4, which contains an array of sixteen driving cells with individual boosting circuits. The individual boosting circuits allow the proposed driver to provide multiple switching current levels by producing only 
the required amount of boosting charge to avoid overshoot. Due to the use of an integrated boosting circuit, the proposed driver does not require external components like the large inductor of $[10,14]$.

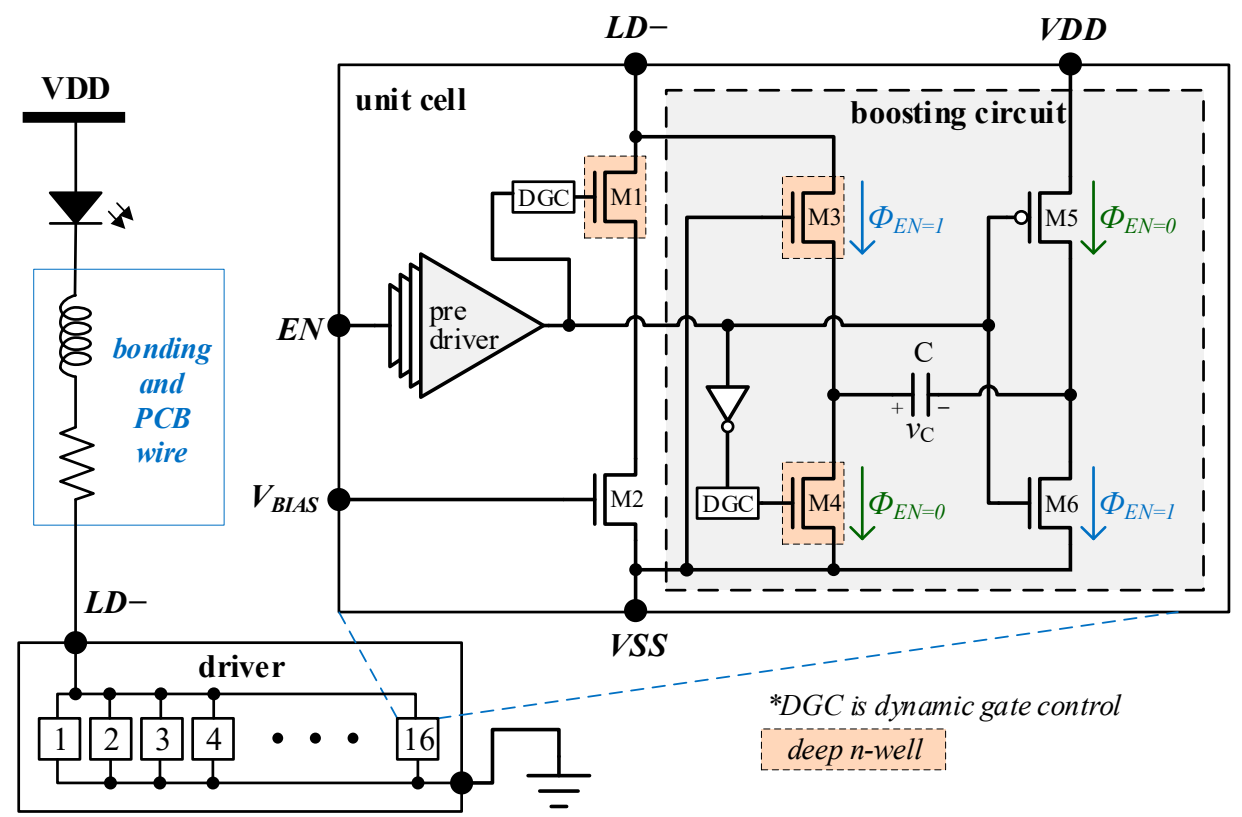

Figure 4. Proposed VCSEL driver with negative voltage boosting circuit.

The boosting circuit provides up to $2 x \mathrm{VDD}$ boosting by generating a short-lived negative VDD using the MOSFETs and the capacitor in the boosting circuit. The circuit operation of the proposed driver can be divided in to three phases as shown in Figure 5, which are pre-charge, boosting and normal driving phases. During the pre-charge phase, the built-in capacitor is charged to VDD in opposite polarity, while no current is supplied to the VCSEL. During the boosting phase, the capacitor is connected to provide negative VDD (-VDD), which can drive the VCSEL rapidly to the target level. During the normal driving phase, the boosting capacitor is disconnected, and thus M1 and M2 flow a fixed amount of current through the VCSEL.

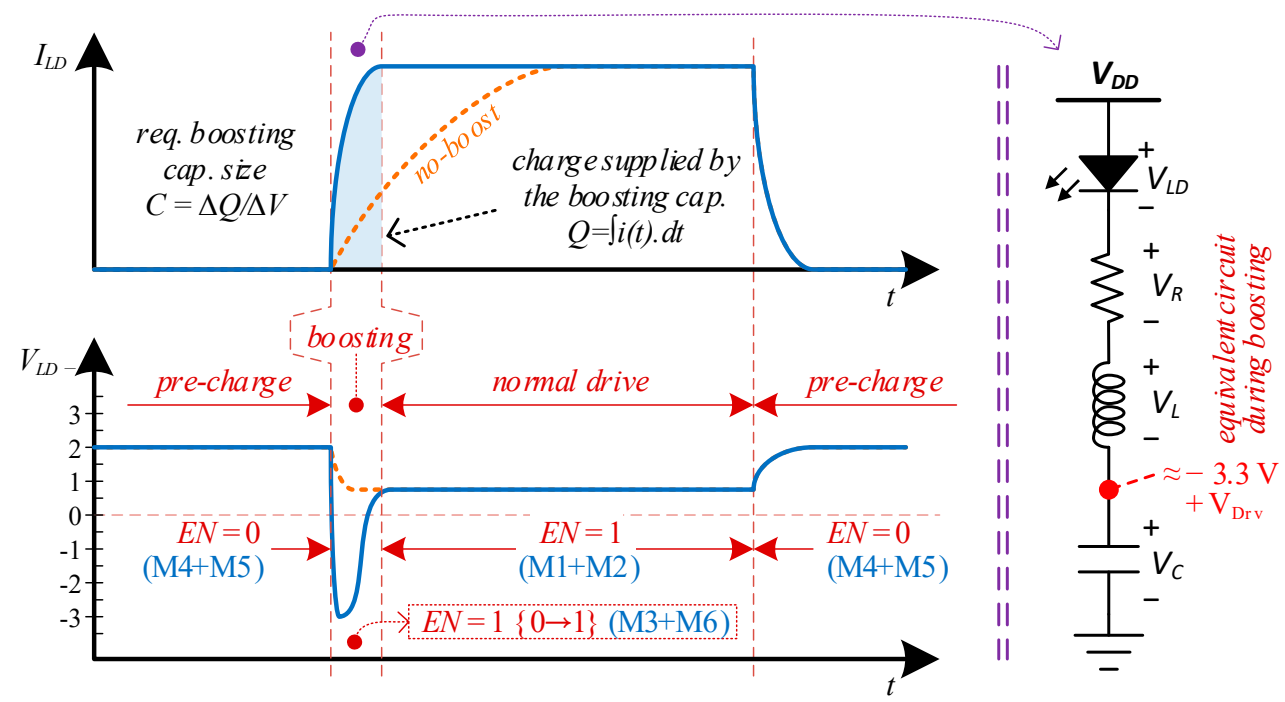

Figure 5. Operational phases and equivalent circuit during the boosting phase (for calculating the amount of charge supplied).

During boosting, the amount of charge supplied (by the boosting capacitor) depends upon the final level of the VCSEL current and the rise time; i.e., $Q=\int i_{L D}(t) \cdot d t$, as shown in Figure 5 . Since a 
short rise time is provided by the proposed driver, the charge supplied by the capacitor during the short rise time is also small. Due to the relation of $C \propto \Delta Q$, a small-sized boosting capacitor is needed in the proposed driver, resulting in small overhead in the area and power consumption. To supply an appropriate amount of charge for sixteen VCSEL current levels, the proposed driver reduces the effective boosting capacitor size when the target current level is low. This is accomplished by distributing small boosting capacitors inside each unit cell instead of one large shared capacitor.

In Figure 4, when EN =0, the boosting capacitor in each unit cell is pre-charged to $-3.3 \mathrm{~V}$, using M4 and M5. When EN switches to 1, M6 connects the negative terminal of the capacitor to ground. The potential on the source of M3 goes to $-3.3 \mathrm{~V}$, causing M3 to turn on and bring LD- to approximately $-3.3 \mathrm{~V}$. The capacitor charge depletes as the VCSEL current rises, causing M3 to turn off when $V_{C}$ is approximately $-550 \mathrm{mV}\left(V_{G S, M 3} \approx 550 \mathrm{mV}\right.$, the threshold voltage $V_{T H}$ of MOSFET in the employed process technology is $550 \mathrm{mV}$ ).

As some of the MOSFETs in the circuit are exposed to negative voltages, there are two issues to be addressed. First, both M4 and M1 are exposed to negative voltage on their drain terminals when they should be off, which causes them to continue to conduct. To overcome this problem, the dynamic gate control presented in [18] is incorporated. Dynamic gate control pulls the gate terminal to negative voltage to keep the MOSFET in the cut-off mode, although the original gate voltage would have been zero. Second, the negative drain voltage causes the bulk-drain pn-junction of MOSFETs to become forward biased. To overcome this problem, a body-switching technique presented in [18] is exploited. The body switching circuit connects the bulk terminal to the lower potential node between drain or source. As the bulk voltage of the MOSFET may change from the ground potential, we choose a CMOS process technology with deep n-well to create an isolated p-well to act as substrate for M1, M3 and M4.

\subsection{Estimating the Size of the Boosting Capacitor}

To estimate the required boosting capacitor size, we can apply the Kirchhoff voltage law (KVL) (during boosting, when M3 and M6 are operational), which gives (6). By solving the 2nd order differential equation of (6) for voltage across the capacitor, we can obtain (7).

$$
\begin{gathered}
V_{R L}=R \cdot i_{L D}(t)+L \cdot \frac{d i_{L D}(t)}{d t}+\frac{1}{C} \int i_{L D}(t) \cdot d t, \\
v_{\mathcal{C}}(t)=e^{-\alpha t}\left\{A_{1} \cos \beta t+A_{2} \sin \beta t\right\}+V_{R L}
\end{gathered}
$$

In (7), $\alpha=R / 2 L$ and $\beta=\sqrt{(R / 2 L)^{2}-4 / L C}$, which are the real and imaginary part of the roots of the characteristic equation. $A_{1}=V_{C 0}-V_{R L}$ and $A_{2}=\left(I_{L D 0} / C+\alpha A_{1}\right) / \beta$ are defined by the initial conditions. When the boosting phase starts, $V_{C 0}$ is the initial voltage on the capacitor and $I_{L D 0}$ is the initial current through the inductor/capacitor (same as the bias current of VCSEL). To ensure maximum utilization of the added boosting circuit, the response of (6) must be under-damped, which can be verified by $\beta^{2}<0$. Similarly, the current through the capacitor (and the VCSEL) can be expressed by $i_{L D}=C d v_{\mathcal{C}}(t) / d t$, which is expanded to (8):

$$
\begin{aligned}
& i_{L D}(t)=C \cdot \frac{d}{d t}\left[\left\{A_{1} \cdot e^{-\alpha t} \cdot \cos \beta t\right\}+\left\{A_{2} \cdot e^{-\alpha t} \cdot \sin \beta t\right\}+V_{R L}\right], \\
& i_{L D}(t)=C e^{-\alpha t}\left[\left(\beta A_{2}-\alpha A_{1}\right) \cos \beta t+\left(\alpha A_{2}-\beta A_{1}\right) \sin \beta t\right],
\end{aligned}
$$

Now, we can plot (7) and (8) to estimate the total required capacitance. For example, Figure 6 shows the current and voltage plot using (7) and (8), for various sizes of the boosting capacitor, targeting the switching current of $1 \mathrm{~A}$ with a bias current of $100 \mathrm{~mA}$. Considering charge-sharing losses in the circuit, initial capacitor voltage $V_{C 0}$ of $-3.2 \mathrm{~V}$ (instead of $-3.3 \mathrm{~V}$ ) is used, which predicts the maximum capacitor size to be $94 \mathrm{pF}$. For the maximum capacitor size, the current and voltage curves reach $1.1 \mathrm{~A}$ and $-550 \mathrm{mV}$, respectively, at nearly the same time. It is important to choose an 
optimal maximum capacitor size, since the boosting circuit automatically disconnects itself only when $v_{\mathcal{c}}(t)>-550 \mathrm{mV}$. If we select too large a capacitor, the current may exceed the target level before the capacitor disconnects at $-550 \mathrm{mV}$, causing overshoot. It is possible, however, to use a smaller boosting capacitor for relaxed rise time requirements. For smaller boosting capacitor sizes, the boosting circuit does not bring the current to the required level, since the capacitor voltage reaches $-550 \mathrm{mV}$ too early, which will cause the rise time to be prolonged.
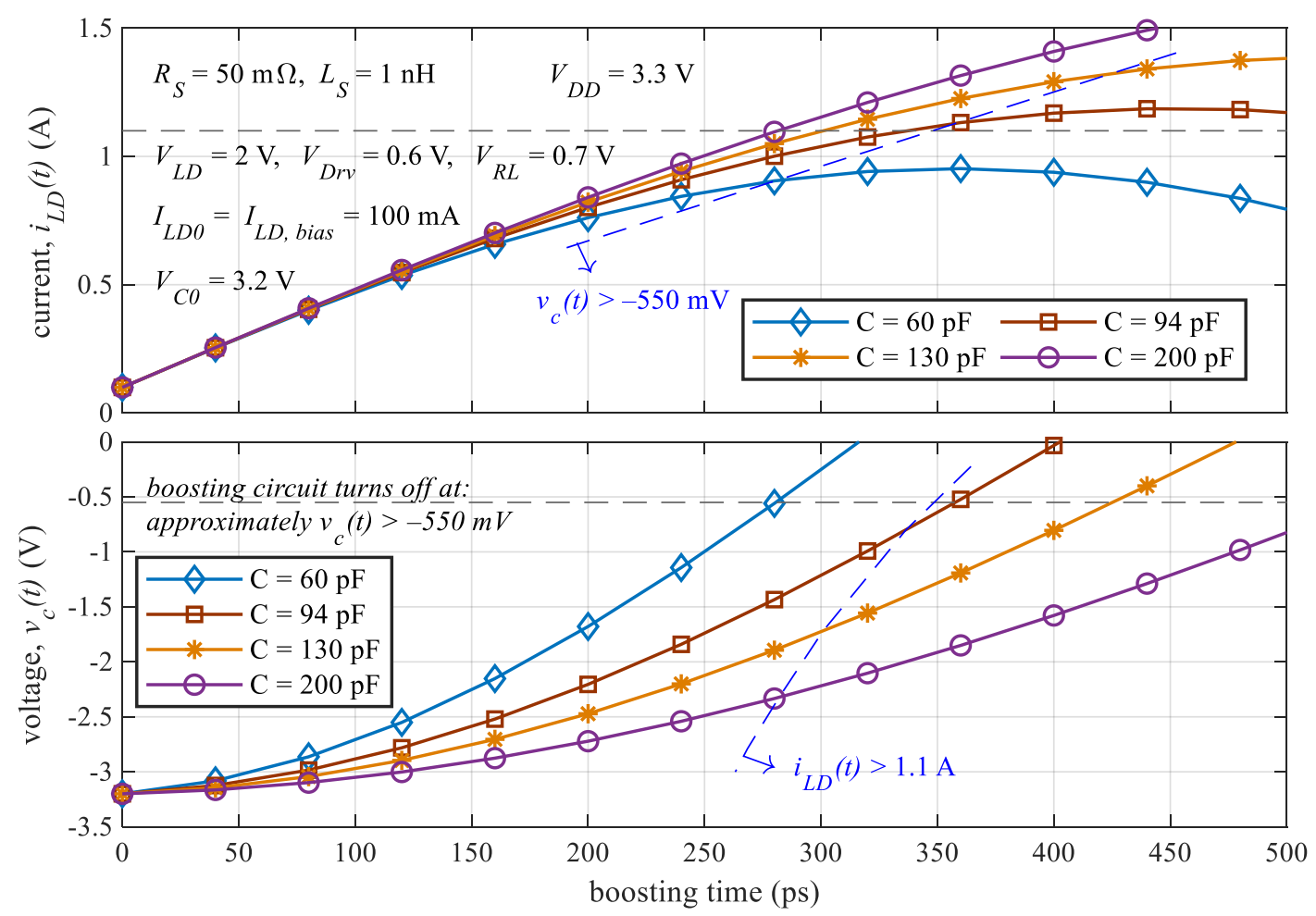

Figure 6. Various drive current transitions using Equation (8) over boosting time for different boosting capacitor sizes. An optimal boosting capacitor selected from this example is $94 \mathrm{pF}$.

\subsection{Design Procedure}

In order to exploit the benefits of the proposed architecture for a given set of constraints $\left(L_{s}, R_{s}, t_{r}, V_{L D}, V_{D r v}, I_{L D, \text { Bias }}\right.$ and $\left.I_{L D, M a x}\right)$, the following procedure can be adapted.

1. Choose nominal VDD level.

2. Calculate the voltage drop across the wire parasitics $\left(L_{S}+R_{S}\right)$, using (2).

3. Plot (7) and (8), for various capacitor sizes.

a. To see the boosting behavior for each capacitor size.

4. Choose the size of the boosting capacitor $C$ from the plot of (7) and (8),

a. Such that $I_{L D}$ curve reaches $I_{L D, M a x}$ when $v_{\mathcal{C}}(t) \approx-V_{T H}$

5. In step 4 , the duration $t_{\text {boost }}$ to reach from $I_{L D, b i a s}$ to $I_{L D, M a x}$ represents minimum achievable rise time, using the current VDD level.

6. If the required rise time $t_{r}>t_{\text {boost }}$,

a. Increase the VDD level and go to step 2.

7. If the required rise time $t_{r}<t_{\text {boost }}$, 
a. Decrease the VDD level and go to step 2 .

8. Implement the circuit in Figure 4 with the chosen boosting capacitor size

a. For $N$ number of unit cells in the driver.

b. Each unit cell must contain boosting capacitor size of $C_{\text {boost }} / N$.

\section{Results}

For comparison, both conventional driver (without boosting circuit) and the proposed driver (with boosting circuit) are designed in 130-nm 3.3 V CMOS process using Cadence Virtuoso and simulated using Cadence Spectre. Both the drivers include 16 switching current cells, allowing configurable switching current strengths up to $1 \mathrm{~A}$ with a resolution of $62.5 \mathrm{~mA}$. The maximum boosting capacitor of $94 \mathrm{pF}$ (pre-determined using the analytical model of (6) and (7)) is divided equally among all the unit cells. The simulation setup realizes a die-to-die bonding scenario of Figure $7 \mathrm{a}$, as shown in Figure $7 \mathrm{~b}$. A piece-wise linear Verilog-A VCSEL model is used for the simulations, targeting VCSELs such as shown in [19].

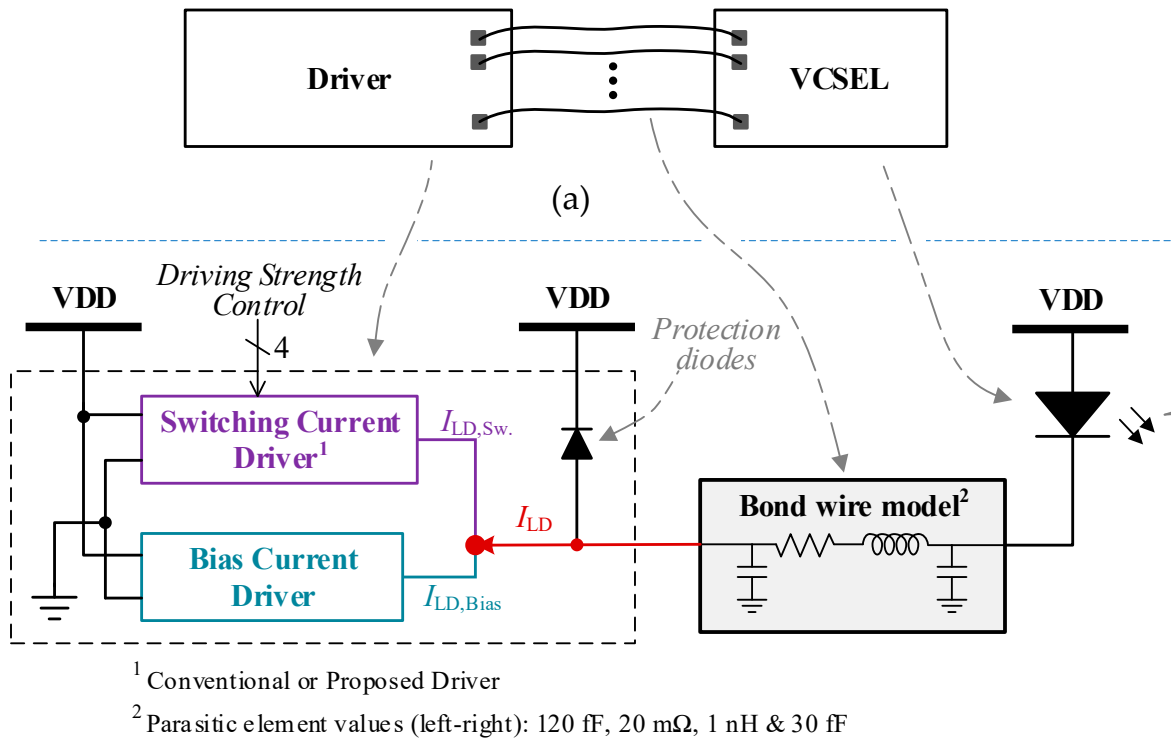

(b)

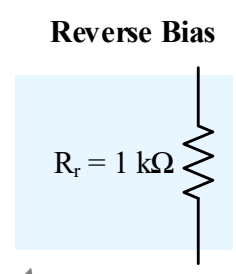

Forward Bias

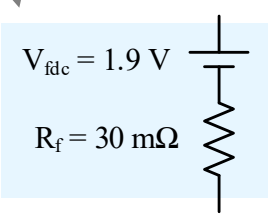

(c)

Figure 7. (a) A typical VCSEL and driver connection using die-to-die bonding, (b) Simulation setup, realization of the typical VCSEL/driver connection, (c) Linear piece-wise model of the VCSEL.

Figure 8 shows the simulation results obtained by operating all 16 unit cells, providing the maximum switching current of $1 \mathrm{~A}$ and the bias current of $100 \mathrm{~mA}$. The conventional driver (blue curve) offers a current rise time of 833 ps, while the proposed driver (red curve) offers 270 ps-a notable reduction of $67.6 \%$. The rise time reduction is achieved at the expense of a $57 \%$ increase in the unit cell area. The voltage waveform of the proposed driver (red curve) shows that the LD- node of Figure 4 is pulled to negative voltage, causing a large voltage-drop across the parasitic elements of the wire, which results in a sharper rise in current. During the falling transition, the voltage $V_{L D-}$ reaches as high as $5 \mathrm{~V}$ in both the conventional and the proposed driver. This is because the parasitic inductance of the wire resists the change in current from $I_{L D, M a x}$ to $I_{L D, \text { Bias }}$ by raising the voltage on the $V_{L D-}$ terminal. Reverse-biased diodes are added between $V_{L D-}$ and VDD to sink the current during the falling transition.

For the above simulations, the VCSEL consumes an average power of $1987 \mathrm{~mW}$, while the boosting circuit consumes $181 \mathrm{~mW}$ (only 10\% overhead), for the target current pulses with a width of $3 \mathrm{~ns}$ and a period of $6 \mathrm{~ns}$ - a typical specification of 3D sensing/ranging applications [20]. On the other hand, to 
achieve a rise time of 270 ps using the conventional driver, a supply of $5.3 \mathrm{~V}$ is needed for the VCSEL, consuming an average power of $3200 \mathrm{~mW}$ ( $47.6 \%$ higher than the proposed technique). The energy consumed per boosting cycle by the boosting circuit and VCSEL is $1.16 \mathrm{~nJ}$ and $11.92 \mathrm{~nJ}$, respectively. When the target specification of the pulse requires larger width or a longer period, the ratio of boosting circuit to VCSEL power consumption can be further reduced.

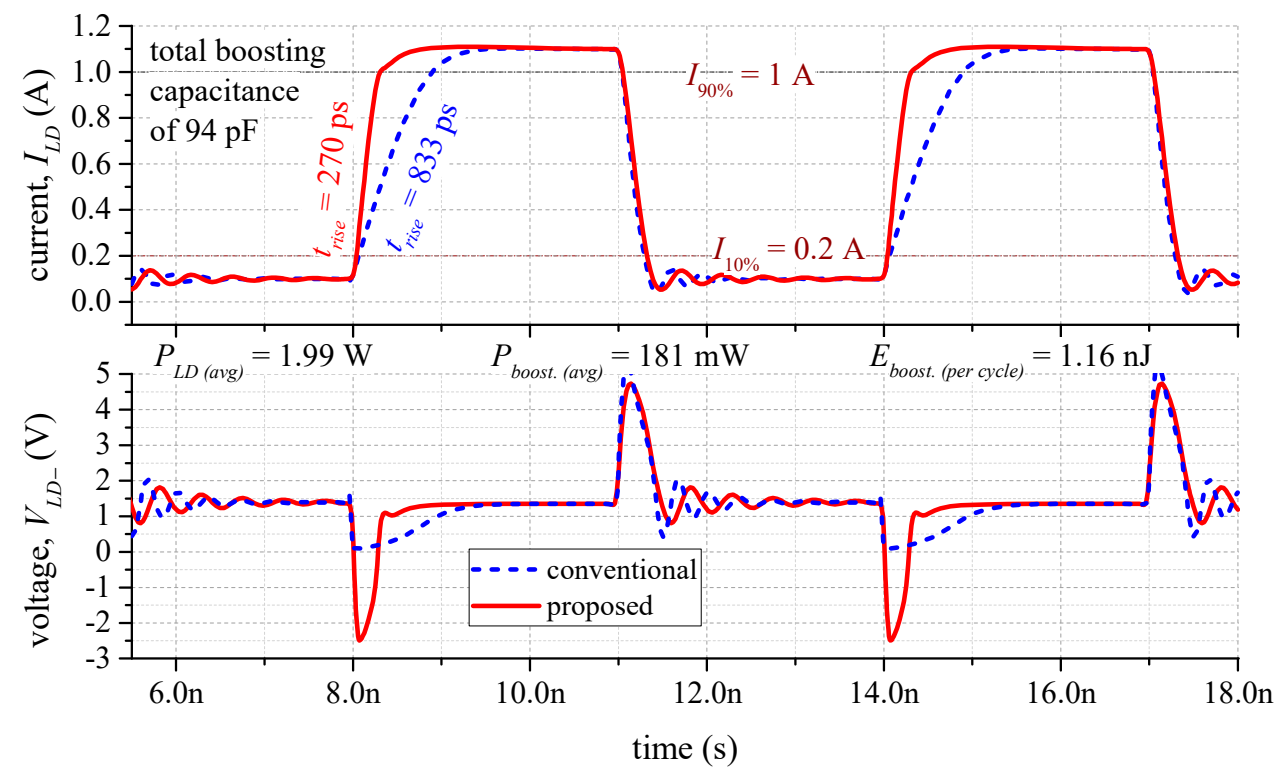

Figure 8. The simulation results of driving current and voltage for the proposed vs. conventional driver.

A second set of circuit simulations is carried out to illustrate the operation of the proposed driver for multiple current levels. Figure 9 shows simulation results when using all 16 cells (16x), 12 cells (12x), and eight cells (8x) of the driving cells to obtain target switching current of $1 \mathrm{~A}, 750 \mathrm{~mA}$ and $500 \mathrm{~mA}$, respectively. Since the boosting capacitance is equally distributed among the driving cells as illustrated in Figure 4, the effective boosting capacitance is determined by the number of active driving cells. Figure 9 demonstrates $12 \mathrm{x}$ and $8 \mathrm{x}$ driving with effective boosting capacitance of $70.5 \mathrm{pF}$ $(94 \mathrm{pF} \times 12 / 16)$ and $47 \mathrm{pF}(94 \mathrm{pF} \times 8 / 16)$, respectively. The reduction in effective boosting capacitance limits the supplied charge to the just needed amount during boosting, which prevents overshoot at lower currents.

\section{Discussion}

The simulation results are summarized in Table 1, which reveals two ways of looking at the benefits provided by the proposed negative voltage boosting technique. Firstly, by employing the proposed technique in a driver, the rise time of the current is reduced, as demonstrated by Table 1 (Proposed vs. Conventional-1). Secondly, using the proposed technique, we can reduce the supply voltage while maintaining the short rise time, reducing the power consumption by $32.25 \%$ compared to the conventional-2 driver. This allows significant reduction in power consumption, as shown in Table 1 (Proposed vs. Conventional-2). When comparing with inductor boosting techniques, the proposed technique provides similar rise times with lower power consumption and no external components. The proposed negative voltage technique, being fully integrated, allows simpler and quick adoption for a wide range of applications. 


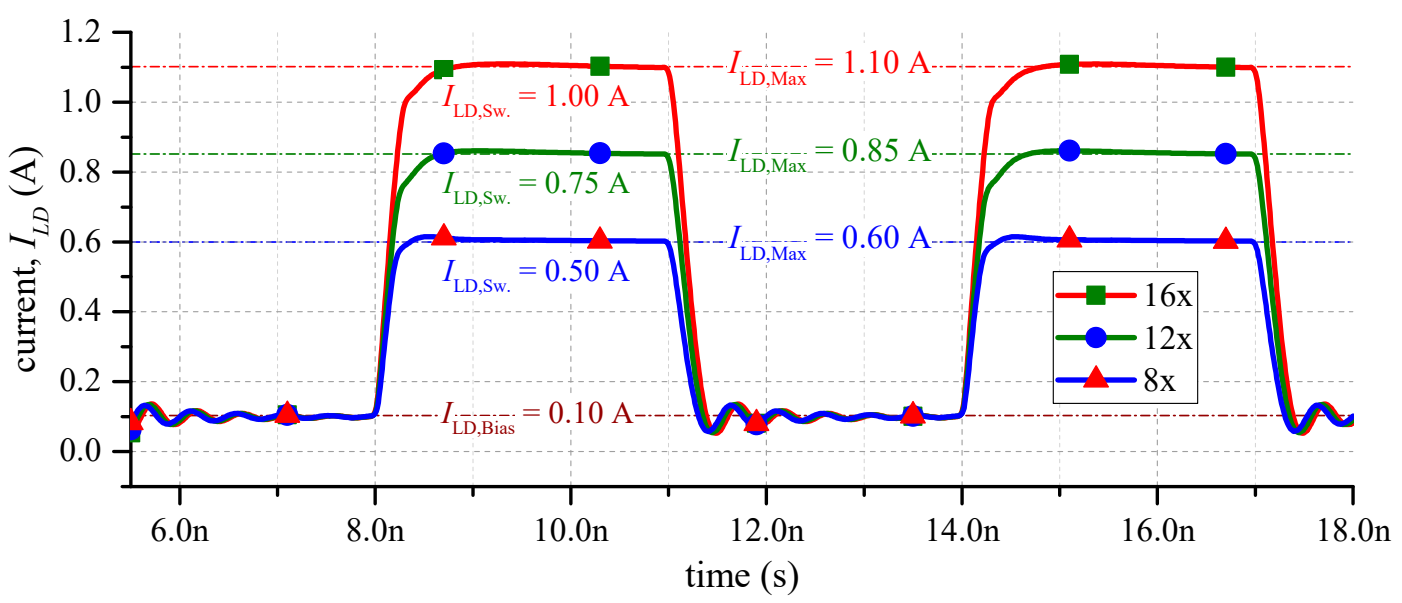

Figure 9. The simulation results of the proposed driver while operating for multiple target current levels.

Table 1. Comparison of the existing techniques with the proposed negative voltage boosting.

\begin{tabular}{|c|c|c|c|c|}
\hline \multirow{2}{*}{ Parameter } & \multicolumn{2}{|c|}{ Conventional } & \multirow{2}{*}{ Inductor Boosting ${ }^{2}$} & \multirow{2}{*}{ Proposed } \\
\hline & 1 & 2 & & \\
\hline Additional wires & No & No & Yes & No \\
\hline External components & No & No & Yes & No \\
\hline Average Power & Low & High & \multirow{2}{*}{ High } & Low \\
\hline Consumption $^{1}$ & $1987 \mathrm{~mW}$ & $3200 \mathrm{~mW}$ & & $2168 \mathrm{~mW}$ \\
\hline Rise Time & Large & Short & \multirow{2}{*}{ Short } & Short \\
\hline Nuse imfle & 833 ps & $270 \mathrm{ps}$ & & $270 \mathrm{ps}$ \\
\hline Area & Small & Small & Medium & Medium \\
\hline
\end{tabular}

${ }^{1}$ During pulse generation; ${ }^{2}$ Estimation using generic implementation.

\section{Conclusions}

The paper presented a capacitor-based negative voltage boosting technique for VCSEL drivers, which provides substantial reduction in the rise time of the VCSEL current. The proposed driver offered up to three times reduction in rise time with less than $10 \%$ additional power consumption. In other words, the proposed technique can choose the minimum supply voltage, while maintaining the rise time, resulting in significant power savings. This same technique can be modified to create positive boosting for driving common-cathode VCSEL/laser diodes. To further reduce power consumption, the energy stored in the parasitic inductor can be used to charge the boosting capacitor during the falling transition of the current.

Author Contributions: Conceptualization, S.A. and S.A.A.S.; methodology, S.A.; formal analysis, S.A.A.S.; investigation, S.A. and S.A.A.S.; writing—original draft preparation, S.A.; writing-review and editing, S.A.A.S.; supervision, H.K.; project administration, H.K.; funding acquisition, H.K.

Funding: This research was supported by Basic Science Research Program through the National Research Foundation of Korea (NRF) funded by the Ministry of Education with grant number [2017R1D1A1B04032098] and by the Center for Integrated Smart Sensors funded by the Ministry of Science, ICT \& Future Planning as Global Frontier Project (CISS-2017). It was also funded by the KIAT (Korea Institute for Advancement of Technology) grant funded by the Korea Government (MSS: Ministry of SMEs and Startups). (No. S2755555, HRD program for 2019).

Acknowledgments: The authors would like to thank ABOV Semiconductor for providing technical support throughout the process of this research work.

Conflicts of Interest: The authors declare no conflict of interest. 


\section{References}

1. Shi, J.; Yin, B.; Qi, N.; Bai, R.; Li, Z.; Hong, Z.; Chiang, P.Y. Design Techniques for Signal Reflection Suppression in High-Speed 25-Gb/s Laser Drivers in CMOS. IEEE Photonics Technol. Lett. 2018, 30, 39-42. [CrossRef]

2. De Marcellis, A.; Palange, E.; Nubile, L.; Faccio, M.; Di Patrizio Stanchieri, G.; Constandinou, T.G. A Pulsed Coding Technique Based on Optical UWB Modulation for High Data Rate Low Power Wireless Implantable Biotelemetry. Electronics 2016, 5, 69. [CrossRef]

3. Ledentsov, N.; Shchukin, V.; Ledentsov, N.; Kropp, J. Design Considerations for Single-Mode Vertical-Cavity Surface-Emitting Lasers with Impurity-Induced Intermixing. IEEE J. Quantum Electron. 2016, 52, 1-6.

4. Liu, A.; Wolf, P.; Lott, J.; Bimberg, D. Vertical-cavity surface-emitting lasers for data communication and sensing. Photonics Res. 2019, 7, 121-136. [CrossRef]

5. $\quad$ Ma, Y.-S.; Lin, Z.-Y.; Lin, Y.-T.; Lee, C.-Y.; Huang, T.-P.; Chen, K.-H.; Lin, Y.-H.; Lin, S.-R.; Tsai, T.-Y. 29.6 A Digital-Type GaN Driver with Current-Pulse-Balancer Technique Achieving Sub-Nanosecond Current Pulse Width for High-Resolution and Dynamic Effective Range LiDAR System. In Proceedings of the IEEE International Solid-State Circuits Conference (ISSCC), San Francisco, CA, USA, 17-21 February 2019; pp. 466-468.

6. Nissinen, J.; Kostamovaara, J. A 4 a peak current and 2 ns pulse width CMOS laser diode driver for high measurement rate applications. In Proceedings of the European Solid-State Circuits Conference (ESSCIRC), Bucharest, Romania, 16-20 September 2013; pp. 355-358.

7. Nissinen, J.; Kostamovaara, J. A High Repetition Rate CMOS Driver for High-Energy Sub-ns Laser Pulse Generation in SPAD-Based Time-of-Flight Range Finding. IEEE Sens. J. 2016, 16, 1628-1633. [CrossRef]

8. Koelle, U.; Johnson, S.R.; Kelkar, P.; Turpin, R.; Dowd, P.; Pan, G.; Zhang, Y.-H. Flip-chip bonding of VCSEL arrays with silicon driver chips for high speed data links. In Proceedings of the IEEE Lasers and Electro-Optics Society (LEOS), 11th Annual Meeting, Orlando, FL, USA, 1-4 December 1998; pp. $166-167$.

9. $10 \mathrm{Gbps} 100 \mathrm{~mA}$ N-Side down LASER Driver for Direct Bonding to LD. Available online: http://www.advico. de/index.php/8-home/2-10-gbps-100ma-n-side-down-laser-driver-for-direct-bonding-to-ld (accessed on 27 September 2019).

10. Zeng, Z.; Sun, K.; Wang, G.; Zhang, T.; Kulis, S.; Gui, P.; Moreira, P. A Compact Low-Power Driver Array for VCSELs in 65-nm CMOS Technology. IEEE Trans. Nucl. Sci. 2017, 64, 1599-1604. [CrossRef]

11. Design and Test of Fast Laser Driver Circuits. iC-Haus GmbH, August 2012. Available online: https://www. ichaus.de/upload/pdf/WP4en_DesignTestFastLaserDriver_08082012.pdf (accessed on 17 October 2019).

12. Glaser, J. High Power Nanosecond Pulse Laser Driver Using an GaN FET. In Proceedings of the PCIM Europe 2018, Nuremberg, Germany, 5 July 2018; pp. 1-8.

13. Tajfar, A.; Zamprogno, M.; Villa, F.; Zappa, F. A 20 A Sub-Nanosecond Integrated CMOS Laser Diode Driver for High Repetition Rate SPAD-Based Direct Time-of-Flight Measurements. In Proceedings of the International Conference on Computing Electronics \& Communications Engineering (iCCECE), Southend, UK, 16-17 August 2018; pp. 272-276.

14. Barnes, E.D.; Singer, L.A.; Weinberg, H.; Analog Devices, Inc. Pulsed Laser Diode Driver. U.S. Patent 10158211B2, 23 March 2017.

15. Lee, J.-Y.; Kim, G.-S.; Oh, K.-I.; Baek, D. Fully Integrated Low-Ripple Switched-Capacitor DC-DC Converter with Parallel Low-Dropout Regulator. Electronics 2019, 8, 98. [CrossRef]

16. Alateeq, A.; Almalaq, Y.; Matin, M. A Performance of the Soft-Charging Operation in Series of Step-Up Power Switched-Capacitor Converters. J. Low Power Electron. Appl. 2018, 8, 8. [CrossRef]

17. Rigault, S.; Moeneclaey, N.; Labrak, L.; O'Connor, I. CMOS VCSEL driver dedicated for sub-nanosecond laser pulses generation in SPAD-based time-of-flight rangefinder. In Proceedings of the Conference on Design of Circuits and Integrated Systems (DCIS), Lyon, France, 14-16 November 2018; pp. 1-6.

18. Ju, Y.-M.; Shin, S.-U.; Huh, Y.; Park, S.-H.; Bang, J.-S.; Kim, K.-D.; Choi, S.-W.; Lee, J.-H.; Cho, G.-H. 10.4 A hybrid inductor-based flying-capacitor-assisted step-up/step-down DC-DC converter with $96.56 \%$ efficiency. In Proceedings of the IEEE International Solid-State Circuits Conference (ISSCC), San Francisco, CA, USA, 5-9 February 2017; pp. 184-185. 
19. 940nm Multi Mode High Power VCSEL Array. II-VI Laser Enterprise. Part\# APS6101010001. Available online: www.ii-vi.com/wp-content/uploads/2019/07/940nm-Multi-Mode-High-Power-VCSEL-Array-web1.pdf (accessed on 17 October 2019).

20. Kostamovaara, J.; Huikari, J.; Hallman, L.; Nissinen, I.; Nissinen, J.; Rapakko, H.; Avrutin, E.; Ryvkin, B. On Laser Ranging Based on High-Speed/Energy Laser Diode Pulses and Single-Photon Detection Techniques. IEEE Photonics J. 2018, 7, 1-15. [CrossRef]

(C) 2019 by the authors. Licensee MDPI, Basel, Switzerland. This article is an open access article distributed under the terms and conditions of the Creative Commons Attribution (CC BY) license (http://creativecommons.org/licenses/by/4.0/). 